\title{
Musik in der Neurorehabilitation nach einem Schlaganfall
}

\author{
F. Speth ${ }^{1} ;$ S. Evers ${ }^{2}$ \\ ${ }^{1}$ Berlin; ${ }^{2}$ Klinik für Neurologie, Krankenhaus Lindenbrunn, Coppenbrügge
}

\begin{abstract}
Schlüsselwörter
Musik, Musiktherapie, Neurorehabilitation, Schlaganfall

Zusammenfassung

In diesem Übersichtsartikel wird der Stand der Forschung zu Effekten und Wirkweisen musiktherapeutischer Anwendungen in der Neurorehabilitation mit einem Fokus auf Schlaganfallrehabilitation dargestellt. Daran anknüpfend werden offene Untersuchungsfelder beschrieben, die sich aus dem zunehmenden Einsatz von robotergestützten Systemen, die klangliche Umgebungen nutzen, ergeben.
\end{abstract}

Keywords

Music, music therapy, neurorehabilitation, stroke

Summary

In this review, the current research regarding effects and mechanisms of music therapy in neurorehabilitation with a focus on stroke rehabilitation is presented. Subsequently, unresolved aspects of research are described which are related to robot-assisted systems using sound surroundings.

Korrespondenzadresse

Dr. Florina Speth

Adalbertstr. 72, 10997 Berlin

florina.speth@gmail.com
Music in neurorehabilitation after stroke

Nervenheilkunde 2018; 37: 410-418

eingegangen am: 10. März 2018

angenommen am: 4. April 2018
Musik wird in der menschlichen Evolution eine phylogenetische Schlüsselrolle zugeschrieben, da sie unsere kognitiven, motorischen und sozialen Systeme stark mitgeformt hat: Musik spezialisierte unser Gehirn darauf, komplexe Verarbeitungsebenen für akustische Reize, Vokalisation, koordinierte Bewegungen sowie feinmotorische Fähigkeiten auszubilden. Des Weiteren prägte Musik die Entwicklung unserer emotionalen Ausdrucks- und Verständnisfähigkeit mit $(3,31,47)$. Seit der Antike findet Musik ihren Einsatz in einer Vielzahl von Kontexten, die von Ritualen, Sport und Kunst bis hin zu Therapie reichen $(2,65)$. Dies mag mitunter daran liegen, dass $\mathrm{Mu}$ sik perzeptuelle, motorische, emotionale und soziale Aspekte stark und gezielt beeinflussen kann (65).

Studien zu Musikanwendungen in therapeutischen Settings zeigten, dass Musik sich positiv auf Rehabilitationsprozesse, Gesundheitszustand und Wohlbefinden auswirken kann $(17,36)$. Gründe hierfür sind unter anderem in der aktivierenden Wirkung von Musik zu finden. Musik kann Bewegungsmuster aktivieren, sie kann Stimmung, Aufmerksamkeit, Entspannung und Erregungszustände beeinflussen, das Gedächtnis trainieren und Auswirkungen auf unser soziales Verhalten in Gruppen haben $(9,31,59,60)$. Während wir Musik hören, uns dazu bewegen, aktiv Musik produzieren oder uns selbiges nur vorstellen, sind große und weitverteilte Areale des Gehirns involviert, die sich auf die Verarbeitung akustischer Reize, emotionaler Evaluation, kognitiver und motorischer Domänen verteilen. Wird eine musikalische Aktivität regelmäßig ausgeübt, fördert dies insbesondere sensomotorische und kognitive Domänen $(7,32,74)$. Neben nachhaltig geformten neuronalen Strukturen, die beispielsweise durch musikalisches Training entstehen, kann Musik unsere Stimmung, Kognition und motorische Perfor- mance auch temporär stark beeinflussen $(2,25,59,60,61)$. Diese Aspekte erklären mitunter, weshalb Musik auch als therapeutisches Werkzeug seit der Antike ihre Verwendung findet $(2,9,10,32)$.

In der Neurorehabilitation kann Musik genutzt werden, um verloren gegangene Fähigkeiten wieder zu erlernen und zu trainieren, um noch vorhandene Fähigkeiten, die durch neurodegenerative Erkrankungen beeinträchtigt werden, aufrecht zu erhalten, und, um motorische, kognitive, psychische oder soziale Defizite zu verbessern $(32,42,47,49,56,73)$.

Ein wachsendes Interesse an der Erforschung von Effekten und möglichen Wirkweisen von Musik spiegelt sich in der seit den 1990er-Jahren beständig zunehmenden Anzahl wissenschaftlicher Untersuchungen zum Einsatz von Musik in der Neurorehabilitation (17). Gebauer und Vuust zeigten in einer Literaturrecherche zu Studien mit dem Wort "music intervention" im Jahr 2014, dass die Anzahl von Publikationen, von 5 im Jahr 1990 auf 225 im Jahr 2000 anstieg. Diese Zunahme der Veröffentlichungen dokumentiert das wachsende Interesse zur Forschung über Musik als therapeutisches Werkzeug, das von der Rehabilitationswissenschaft, der Musiktherapie als eigenständiges Forschungsgebiet bis hin zur Musikwissenschaft, Neurologie, Medizin und Technik reicht. Eine aktuelle Literaturrecherche mit den Stichworten „music + adverse effects“, „music intervention + side effects", , music intervention + or therapy + or adverse effects/ side effects" zeigte, dass Studien entweder keine oder positive Effekte von Musik beschreiben. Die Studien, die Probleme zu Musikanwendungen aufzeigen, beziehen sich auf spezielle Pathologien wie musikogene Epilepsie, Amusie oder Hörstörungen (29). Positive Effekte von Musik als Therapiewerkzeug werden intensiv diskutiert und begründen damit ihre breite Anwendung: 
Wird Musik als Ersatz für ein Medikament eingesetzt, birgt sie den Vorteil, keine Intoxikation zur Folge haben zu können. Musik wird als Stimulanz beschrieben, die neurochemische Prozesse auslöst, die als angenehm empfunden werden und das Belohnungszentrum stimuliert. Beispielsweise dient die Anwendung von Entspannungsmusik im operativen Bereich als Ersatz für Schmerzmittel $(17,70)$. Nahezu die gesamte Patientenpopulation kann von Musik profitieren. Das Spektrum der Studien zu Musikanwendungsfeldern umfasst Persönlichkeitsprobleme, kognitive, motorische, soziale und/oder emotionale Defizite sowie Settings, in denen Musik als Entspannungsinstrument bei Operationen sowie als Instrument zur Steigerung von Aufmerksamkeit, Konzentration und Gedächtnis dient. Dies basiert mitunter auf der Tatsache, dass schon die sehr einfache Aktivität des Musikhörens ein weitverteiltes Gehirnnetzwerk aktiviert, das kognitive, motorische und emotionale Bereiche involviert (61). Bei musikalischen Aktivitäten ist ein verteiltes Gehirnnetzwerk aktiv, das einen Strom akustischer Signalen als Musik mit Bedeutung kodiert. Dies erlaubt es uns, eine bekannte Melodie mitsummen und ohne Anstrengung den Rhythmus mitklopfen zu können, während der aktuelle Erfahrungsraum mit Erinnerungen an die bereits gehörte Melodie verbindet $(2,32,59)$. Musik kann Gefühle, die von Traurigkeit bis hin zu Melancholie, Glück und Genuss reichen, auslösen $(27,28,44)$.

Zusammenfassend verdeutlichen die angeführten Aspekte, dass Gedächtnis, Emotion, motorische und imaginäre motorische Prozesse beim Umgang mit Musik involviert sind. All diese können als therapeutische Treiber genutzt werden: Musik kann gehört werden, für Tanz und Bewegung eingesetzt werden sowie gesungen oder mit Instrumenten angewendet werden $(60,62)$.

Derzeit werden zunehmend neue Technologien, wie beispielsweise Rehabilitationsroboter, in Therapiekontexten verwendet, die Multimedia beinhalten. In diesen Szenarien, wird Klang und Musik oftmals eingesetzt, um virtuelle Realität mit klanglich physischem Realismus anzureichern (55). Klang und Musik werden in diesem Zusammenhang häufig mit der Intention angewendet, die Motivation zu erhöhen und das Trainingsumfeld anzureichern. Insbesondere in diesem Bereich ist noch eine Risikoanalyse erforderlich, die überprüft, ob Klang und Musik für alle Patientenpopulationen und im vorgegebenen Kontext von Nutzen sind. Der Grund für die breite Anwendung von Klang und $\mathrm{Mu}$ sik in neuen Technologieumgebungen für Therapien könnten möglicherweise auf den Ergebnissen der aktuellen Forschung beruhen, die oben zusammengefasst wurden. Diese Ergebnisse stellen Musik als einen Einflussfaktor dar, der sich entweder als unwirksam oder nur therapeutisch wirksam erweisen kann. Wie sich Musik im Zusammenspiel mit der vielseitigen Technologieumgebungen und speziellen Patientenpopulationen auswirkt, ist jedoch offen. Um negative Nebenwirkungen zu vermeiden und um insbesondere die positiven Behandlungseffekte zu erhöhen, sind klinische Untersuchungen erforderlich, die Informationen über eine adäquate Anwendung von Klang oder Musik liefern, die Motivation und Funktion positiv beeinflussen.

\section{Musik in der Schlaganfall- rehabilitation}

Musik und Klang werden für die Rehabilitation nach einem Schlaganfall in den Bereichen des motorischen und kognitiven Trainings, des Sprech- und Stimmtrainings, des psychologischen Trainings, zur Förderung von Entspannung, Interaktion und Krankheitsmanagement eingesetzt (2, $8,17)$. Generell wurden klinisch relevante Ergebnisse zur Wirksamkeit von Musik und Klang für die Rehabilitation nach einem Schlaganfall für motorische Therapien, für kognitives Training und für Musikhören im Zusammenhang mit Stimmungsstörungen gezeigt $(2,8,17,54,60)$. Auch die musikalische Intonationstherapie, ein Training für Aphasie-Patienten, gilt als vielversprechender Ansatz (2). Im folgenden Abschnitt werden die wichtigsten Studien zu Musikinterventionen, die klinisch relevante Ergebnisse in

- motorischem Training,

- kognitivem Training und

- psychologischem Training in der Schlaganfallrehabilitation erzielt haben, zusammengefasst.

\section{Motorik}

Im Bereich der motorischen Schlaganfallrehabilitation wurden verschiedene musikalische Therapietechniken entwickelt, die insbesondere darauf zielen, Gang- und Armfunktion zu verbessern. Seit den späten neunziger Jahren wurde eine Reihe von Studien zu rhythmisch akustischer Stimulation, kurz RAS, durchgeführt. RAS wurde als reproduzierbares Trainingsprotokoll und als artikuliertes Therapieverfahren für Schlaganfall-Patienten mit motorischen Defiziten etabliert. Im RAS-Training wird ein Metronom oder stark rhythmische $\mathrm{Mu}$ sik verwendet, um eine klangliche Umgebung zu bieten, die zur Synchronisation von Bewegungen entlang eines Beats anregen. RAS wurde klinisch mit SchlaganfallPatienten, die an einer Halbseitenlähmung leiden in Gangtraining, in einseitigem Armfunktionstraining und in bilateralem Armtraining evaluiert (65). In beiden Bereichen, dem Training der oberen und unteren Extremitäten, wurden moderate Effekte auf die Motorik erzielt (8): Bradt et al. 2010 führten einen Cochrane-Review zur Musiktherapie bei erworbener Hirnschädigung durch. Eines der zentralen Ergebnisse dieser Review war, dass RAS-Gangtraining für Schlaganfall-Patienten positive Wirkung zeigt. Diese Schlussfolgerung basierte auf zwei Studien mit geringem Risiko für Bias-Scores, die das RAS-Gangtraining mit insgesamt 98 Schlaganfall-Patienten berücksichtigte. Beide Studien zeigten, dass das RAS-Gangtraining signifikante Verbesserungen in der Schrittlänge, Schrittsymmetrie, Schrittfrequenz und Ganggeschwindigkeit im Vergleich zur physiotherapeutischen Standardbehandlung erzielte (8). Eine weitere klinische Studie wurde durchgeführt, die temporäre Effekte von RAS während eines etablierten Armfunktionstrainings evaluierte. Die Ergebnisse dieser Studie zeigten, dass eine signifikante Abnahme der kompensatorischen Rumpfbewegung, eine Zunahme des Schulterbewegungsraums in der Beugung, und eine leichte Zunahme der Ellenbogenstreckung während des Trainings erreicht wurden. Darüber hinaus verbesserten sich Bewe- 
gungstiming und Bewegungsgeschwindigkeit (65). Malcom et al. führten eine Pilotstudie mit fünf Schlaganfall-Patienten durch, die von einer chronischen Armparese betroffen waren. Sie untersuchten Effekte eines 2-wöchigen Trainingszyklus funktionalen Armtrainings, welches mit RAS kombiniert wurde. Die Studie zeigte, dass sich die Größe des Bewegungsraums verbesserte, kompensatorische Rumpfbewegungen abnahmen, der Schulterbewegungsraum sowie der Bewegungsraum für die Ellbogenstreckung signifikant zunahmen (40). Auch Bewegungstiming und -geschwindigkeit verbesserten sich im Vergleich zum Behandlungsbeginn. Eine weitere Pilotstudie wurde von Whitall et al. durchgeführt. Diese Autoren untersuchten die Auswirkungen eines bilateralen Armtrainings mit rhythmischem Cueing (BATRAC). Nach sechs Wochen BATRACTraining verbesserte sich die motorische Leistung, isometrische Kraft und die Größe des Bewegungsraums. Sowohl die Studie von Malcom et al., als auch die Studie von Whitall et al. hatten keine Kontrollgruppen zum Vergleich, weswegen die Aussagekraft über die Therapieeffekte noch schwach ist. Paul et al. untersuchten die Auswirkungen des aktiven Musizierens auf Ellenbogenstreckung und Schulterbeugung bei 10 Schlaganfall-Patienten. Sie stellten in Folge der Behandlung keine signifikanten Veränderungen fest $(8,48)$. Schneider et al. führten eine klinische Studie mit 20 Patienten durch, die von einer moderaten Armparese betroffen waren und untersuchte Effekte von „Music Supported Therapy“ (MST), einer aktiven Form der Musiktherapie mit Musikinstrumenten. Bei den untersuchten MST-Interventionen erlernten die Patienten leichte Melodien auf einem Keyboard oder einfache Trommelrhythmen auf einem elektronischen Drumpad. Alle Studienteilnehmer erhielten 15 Trainingseinheiten, die zusätzlich zur konventionellen Behandlung durchgeführt wurden. Die Ergebnisse dieser Studie zeigten signifikante Verbesserungen in Geschwindigkeit, Präzision, Gleichmäßigkeit („smoothness") der Bewegungen und Verbesserungen in Tests zu Aktivitäten des täglichen Lebens (62). Eine weitere Studie wurde von Amengual et al. mit 20 Schlaganfall-Patienten durchgeführt, die 4 Wochen lang MST erhielten.
Diese Patienten wurden vor und nach der Behandlung einer transkraniellen Magnetstimulation unterzogen, um Veränderungen in der Erregbarkeit des motorischen Kortex festzustellen (1). Die Ergebnisse zeigten, dass MST-Training neuroplastisch unterstützend wirkt und zur Entwicklung neuer Nervenbahnen führte. Das neu gewachsene Netzwerk wird als auditorisch-sensomotorisches Netzwerk beschrieben. Dieses Netzwerk wurde auch von Rojo et al. beschrieben, die eine Studie zur MST mit bildgebenden Verfahren durchführten (2). Zusammenfassend zeigen die Studien zu MST auf, dass MST die neuronale Plastizität in einem auditorischsensomotorischen Netzwerk fördert. Friedman et al. führten eine Studie durch, um die Wirkung eines interaktiven Musikhandschuhtrainings mit der eines konventionellen Therapieverfahrens und isometrischem Aufgabentraining bei 15 Patienten mit mäßiger Armparese zu vergleichen. Die Ergebnisse zeigten, dass das Musikhandschuhtraining, im Vergleich zu den beiden anderen Interventionen, zu einer stärkeren Verbesserung der Handfunktion führte. Außerdem waren die Patienten, die mit dem Musikhandschuh trainierten, motivierter als die beiden anderen Gruppen (15). Eine ähnliche Wirkung auf die Motivation wurde als Ergebnis einer Studie von Taheri et al. angegeben, in der ein robotischer Handfunktionstrainer mit einem ähnlichen musikalischen Videospiel bewertet wurde (64). Eine weitere klinische Studie zu Effekten von robotergestützem Handfunktionstraining in Kombination mit Musik führte Speth durch. In dieser Studie wurden Effekte dieser Behandlung auf Funktion und Motivation mit robotergestütztem Handfunktionstraining ohne Musik verglichen. Ergebnisse dieser Studie zeigten, dass Musik für schwer betroffene Patienten mit einer stark ausgeprägten Spastik in der Hand zwar die Motivation verbesserte, die Funktion, im Vergleich zu Trainingseffekten ohne Musik, signifikant verschlechterte. Hingegen profitierten mittel bis leicht betroffene Patienten sowohl in Motivation als auch in Funktion signifikant im Vergleich zur Gruppe, die ohne Musik trainierte (63).

\section{Kognition}

Im Bereich des kognitiven Trainings führten Särkämö et al. eine einfach verblindete, randomisierte kontrollierte Studie mit 60 Schlaganfall-Patienten in der sehr frühen Phase nach dem Schlaganfall durch. Die Studie untersuchte, ob das Hören von selbstgewählter Musik, von Hörbüchern oder gar keiner zusätzlichen Aktivität zu Veränderungen in der kognitiven Rehabilitation, der Stimmung und der wahrgenommenen Lebensqualität führt. Die Patienten wurden randomisiert einer Musikhörgruppe, einer Hörbuchhörgruppe oder einer Gruppe zugeordnet, die keine zusätzliche Aktivität erhielt. Alle Studienteilnehmer wurden zu Studienbeginn, nach drei undnach sechs Monaten untersucht. Die Ergebnisse der Studie zeigten, dass sich die Musikhörgruppe, im Vergleich zu den beiden anderen Gruppen, im verbalen Gedächtnis und in der Aufmerksamkeit signifikant verbesserte. Außerdem war die Musikhörgruppe nach sechs Monaten weniger von Depressionen (post-stroke depression), negativer Stimmung und Verwirrungszuständen betroffen. Die Autoren folgerten, dass Musikhören ein weitverbreitetes Gehirnnetzwerk aktiviert, das Areale beider Hemisphären involviert, die mit Aufmerksamkeit, semantischer Verarbeitung, Gedächtnis, motorischen Funktionen und Emotion in Verbindung stehen und dadurch auch weitverteilt neuronale plastische Veränderungen fördern kann. Dies wirke sich wiederum positiv auf die Genesung und die Stimmungslage aus (60). Generell können Stimmungszustände als kritischer Einflussfaktor für Motivation zur Rehabilitation angesehen werden. Gesundheitsexperten betrachten Motivation als sehr wichtige Determinante für positive Rehabilitationsergebnisse (38).

\section{Psyche}

Im Bereich psychologisch relevanter Effekte von Musikanwendungen in der Schlaganfallrehabilitation zeigten die beiden bereits unter dem Punkt Kognition vorgestellten Studien von Särkämö et al. und Friedman et al. sowie auch eine Studie von Raglio et al., dass Musikhören einen positiven Einfluss auf Stimmung, Emotion und 
Motivation bei neurologischen Patienten hat $(15,61,54)$. Dieser Aspekt ist von groBer Bedeutung, da 40\% aller SchlaganfallPatienten nach einem Schlaganfall an einer Depression leiden. Depressive Symptome können sich negativ auf die funktionale Verbesserung, Therapiecompliance, Motivation, die wahrgenommene Lebensqualität, und auf das Sterberisiko auswirken (54). Raglio et al. führten eine narrative Review über die Auswirkungen des Musikhörens als musiktherapeutische Intervention für neurologische Patienten durch und fanden heraus, dass die meisten Studien darauf hindeuteten, dass stimmungsbezogene, depressive Syndrome und Lebensqualität durch Musiktherapie positiv unterstützt wurden.

\section{Wirkmechanismen von Musik}

Laut der Zusammenfassung von Studien, die die Wirksamkeit von Musikinterventionen belegen, kann Musik im Rahmen von Rehabilitationsprozessen in der Schlaganfalltherapie Kognition, Emotion, Motivation und Motorik positiv beeinflussen. Im vorherigen Abschnitt wurden musikalische Interventionen für die Rehabilitation nach einem Schlaganfall zusammengefasst. Diese werden mit der Absicht eingesetzt, kognitive, motorische und psychische Funktionen, soziale oder motivationale Aspekte zu fördern. In diesem Abschnitt werden musikinduzierte Effekte und zugrundeliegende Mechanismen der motorischen Rehabilitation nach dem Schlaganfall beschrieben. Die bisher am besten belegten musikgestützten Effekte in der Rehabilitation nach einem Schlaganfall wurden für RAS-Gangtraining und für Musikhören gezeigt $(8,60)$. Zuerst werden neuroplastische Prozesse beschrieben, die mit musikalischen Aktivitäten und Rehabilitationsprozessen verbunden sind. Dann werden die Auswirkungen des Musikhörens auf Emotionen und Kognition mit einem Fokus auf die Auswirkungen auf die Schlaganfallrehabilitation betrachtet. Zum Abschluss wird der zugrunde liegende Mechanismus von RAS diskutiert.

Musikalische Aktivitäten wie das Synchronisieren von Bewegungen zu einem
Beat, das Spielen eines Instruments oder das Hören von Musik führen zu langanhaltenden neuroplastischen Veränderungen $(1,74)$. Studien zu aktivem Musizieren zeigten bereits, dass musikalische Aktivitäten neuronale Bahnen grundlegend verändern, besonders wenn das musikalische Training bereits in der Kindheit mit hoher Intensität durchgeführt wird $(22,23)$. Auch bei erwachsenen Amateurmusikern wurden bereits neuroplastische Veränderungen belegt (4). Studien, in denen Gehirne von Musikern und Nichtmusikern miteinander verglichen wurden, zeigten, dass hirnanatomische und neuroplastische Veränderungen auftreten können $(4,16,43,22,23)$. Diese Studien zeigten, dass zwischen Musikern und Nichtmusikern neuroplastische Unterschiede im Gehirn und Unterschiede im kortiko-spinalen Trakt existieren. Die Gründe für diese Unterschiede könnten auf trainingsinduzierten neuroplastischen Prozessen beruhen $(2,22,23)$. Wenn musikalisches Training zu neuroplastischen Veränderungen mit langanhaltender Wirkung führt, liegt es nahe, diesen Effekt für Rehabilitation nutzbar zu machen, um das Lernen zu fördern und das Wachstum neuer neuronaler und kortikospinaler Netze zu fördern.

Ein Beispiel für eine aktive Musikintervention, die sich konkret auf dieses Konzept bezieht, ist die „Music Supported Therapy" (MST), bei der das Spielen eines Musikinstruments als motorisches Training dient $(1,62)$. Eine weitere Studie über die Auswirkungen von MST wurde von Van Vugt et al. durchgeführt. In dieser wurde geprüft, ob das akustische Feedback die verantwortliche Komponente innerhalb der MST (in diesem Fall das Spielen bekannter Klavierstücke) ist, die positive Effekte hervorruft. Dazu wurden zwei Gruppen verglichen, die entweder mit normalem oder mit verzögertem akustischem Feedback trainierten (72). Die Ergebnisse zeigten, dass in beiden Gruppen positive Effekte erzielt wurden. Im Rahmen des Nine Hole Peg-Tests (NHPT) und des Tests zur Ermittlung der Tempovariabilität bei Klopfen zu einem Metronom, übertraf die Gruppe, die ein Training mit verzögertem akustischem Feedback erhalten hatte, die andere Gruppe. Da in beiden Gruppen eine Erhöhung der Klopfgeschwindigkeit, ei- ne Verringerung der Klopfvariabilität, bessere Ergebnisse nach der Behandlung im NHPT und eine Erhöhung der Motivation festgestellt wurde, schlossen Vugt et al., dass akustisches Feedback nicht die tragend wirksame Komponente von MST ist, die die positive Auswirkungen auf Funktion und Motivation hat. Die Autoren diskutierten anstelle dessen, ob propriozeptives und visuelles Feedback, trainingsinduzierte neuronale Plastizität und Motivation eines musikalischen Trainings mit vertrauter Musik die tragenden Gründe für Verbesserungen in Funktion und Motivation sein könnten.

Die Studie von Särkämö et al. zeigte, dass Musikhören unter anderem die Stimmung von Schlaganfall-Patienten, in der frühen Phase nach dem Insult, verbessert. Dies deutet darauf hin, dass das Hören von Musik den emotionalen Zustand der Patienten konkret beeinflusst. Die zugrunde liegenden Mechanismen dafür könnten in neurochemischen Prozessen liegen, die durch das Musikhören hervorgerufen werden, was wiederum die Wahrnehmung einer positiven Stimmung bewirken könnte. Chanda et al., die eine Untersuchung zur Neurochemie der Musik durchführten, zeigten, dass das Hören von angenehmer Musik in einer gesunden Studienpopulation motivierend und belohnend wirken kann oder Freude bereitet. Das Hören von Entspannungsmusik kann die Erregungsund Stresslevels beeinflussen. Gemeinsames Musizieren kann die soziale Zugehörigkeit fördern. Darüber hinaus stellten die Autoren positive Auswirkungen von Musik auf das Immunsystem fest (10).

Eine Review von Jäncke zum Verhältnis von Musik, Erinnerung und Emotion weist darauf hin, dass das Hören von Musik Emotionen hervorruft und dabei das limbische System aktiviert wird. Laut Jäncke mangelt es an Untersuchungen, die Emotion, Musik und Gedächtnis in Beziehung setzen und das limbische System somit berücksichtigen. Eine weitere Studie zu diesem Thema von Eschrich et al. untersuchte den Einfluss von Emotionen auf das episodische Langzeitgedächtnis für Musik (14). Eschrich et al. führten eine Studie durch, in der die Auswirkungen struktureller Aspekte eines Musikstücks auf die Gedächtnisleistung, genauer von "emotionalen“ und 
„Weniger emotionalen“ Musikstücken, miteinander verglichen wurden. In dieser Studie wurden die Teilnehmer angeleitet 40 musikalische Ausschnitte hinsichtlich Valenz und Erregung zu bewerten. Eine Woche später wurde eine Wiedererkennungsaufgabe durchgeführt. Die Studie zeigte, dass Musikstücke, die als „positiv“ bewertet wurden, besser in Erinnerung blieben als die anderen Stücke und, dass die eigene Bewertung hierbei eine wichtige Rolle für das episodische Langzeitgedächtnis der Musik spielt. Außerdem wurde festgestellt, dass starke Emotionen, die während des Musikhörens erlebt wurden, die Erinnerung an das Stück mitformen (14). Bei Musikhören im Rahmen der Schlaganfalltherapie müssen diese Aspekte in Bezug auf die therapeutischen Ziele sorgfältig berücksichtigt werden. Wenn der Fokus der Therapie auf der Aktivierung autobiografischer Erinnerungen liegt, können bekannte Musikstücke ein geeignetes Mittel sein. Wenn der Anwendungsgrund von Musik in der Förderung von Motivation liegt, ist zu beachten, dass dies auch unerwünschte emotionale Reaktionen und Ablenkung von der eigentlichen Trainingsaufgabe auslösen kann, da das Erinnern in den Vordergrund tritt. Deshalb ist es sehr wichtig, dass die Musikstücke, die therapeutisch verwendet werden, in Bezug auf definierte Therapieziele sowie im ständigen Austausch mit dem Patienten zu wählen sind. Eine pragmatische Lösung hierfür wäre es, die musikalischen Vorlieben des Patienten abzufragen und ähnliche, aber dem Patienten noch unbekannte Musikstücke, einzusetzen. Eine andere Möglichkeit wäre, Musik zu verwenden, die für den Patienten neu ist, angenehm empfunden wird und dies rückgemeldet wird.

Keller und Rieger zeigten, dass die einfache Aktivität des Musikhörens Bewegungsdrang auslösen kann. Dieses Phänomen ist ein sehr wichtiger Aspekt bei der Betrachtung von Musik in Verbindung mit Motivation: Da das Musikhören auch motorische und kinästhetische Simulationen hervorruft, könnte es relevant sein, zu untersuchen, ob die Motivationslevel von dem musikalischen Material abhängen, das es erlaubt, Bewegungen in Bezug auf seine Struktur zu simulieren. Wenn man an rhythmische Musik denkt, ist es wahr- scheinlich sehr einfach, sich eine Bewegung vorzustellen, die mit dieser Struktur verbunden ist. Wenn eine Kette von Ereignissen wiederholt auftritt, wie es bei einfachen rhythmischen Strukturen der Fall ist, ist es leicht Muster zu erkennen und vorherzusagen. Dies löst eine positive Reaktion unseres Belohnungssystems aus (20). Laut Huron ist die Antizipation einer der Schlüsselmechanismen, die uns auf vorhersagbare Ereignisse vorbereitet und uns ermöglicht energieeffizient und adäquat $\mathrm{zu}$ reagieren (20). Die erfolgreiche Vorhersage zukünftiger Ereignisse sowie die Ausführung einer effektiven und passenden Reaktion wirken sich positiv auf unser Belohnungssystem aus. Wir reagieren schnell, energieeffizient und verwenden nur bei unerwarteten Ereignissen größere Energien auf. Im Falle von RAS ist die Vorhersage des nächsten Ereignisses mit einer bestimmten Bewegung gekoppelt. Verläuft diese Kopplung erfolgreich, aktiviert dies unser Belohnungssystem.

Rhythmus beeinflusst unsere motorischen Reaktionen und formt Bewegungsqualitäten auf niedrigen Verarbeitungsebenen, eventuell spinalen Ebenen, und auf unbewussten Reaktionsebenen mit $(57,58$, 67). Schon Einstein sagte: „Wenn einer mit Vergnügen $\mathrm{zu}$ einer Musik in Reih und Glied marschieren kann, dann hat er sein großes Gehirn nur aus Irrtum bekommen, da für ihn das Rückenmark schon völlig genügen würde." Der Prozess der musikalischen Rhythmusverarbeitung umfasst mehrere Teilaufgaben, wie die Kodierung grundlegender Zeitabstände zwischen Ereignissen und die Intensitätsunterschiede zwischen den Einheiten, die Erkennung von Phrasen, die man auch als mentale Gruppierung bezeichnen kann, die Zuordnung verketteter Einheiten zu einem Muster, und die Wahrnehmung eines Beats, einem Schwerpunkt des Musters, der oft mit einem synchronen Bewegungsimpuls einhergeht (24). Die Fähigkeit, sich rhythmisch oder im Takt zu bewegen, reift meist im Alter von 4-5 Jahren. Dieses Phänomen ist in jeder Kultur zu beobachten (12, 47, 49). Dieser Prozess wird als kognitiv nicht aufwändig beschrieben (39). Rhythmusverarbeitung involviert ein weitverzweigtes Netzwerk, das motorische Planungsregionen wie den prämotorischen Kortex, den
Motorkortex, die Basalganglien und das Kleinhirn umfasst $(47,58,72)$. Der zugrunde liegende Mechanismus von RAS, die Ausrichtung einer Bewegung zu einer rhythmischen Struktur, lässt sich am besten durch das Konzept des „Entrainments“ erklären. Das Konzept des Entrainments geht auf den niederländischen Physiker Christian Huygens zurück, der im Jahre 1666 das Zusammenspiel zweier unabhängiger Uhren mit unterschiedlichen Pendelfrequenzen auf einer gemeinsamen Oberfläche beobachtete (56). Nach einiger Zeit schwangen diese Uhren aufgrund von Modulationen, die von der gemeinsamen Schwungfläche übertragen wurden, synchron. Bei RAS führt der Entrainmentprozess zur Synchronisation von Gehirn, Körper und Umgebung, wobei die Umgebung ein akustisch-rhythmisches Signal trägt. Diese Kopplung von Gehirn und Verhalten wird auch als „Neuro-Entrainment" bezeichnet und beschreibt die zeitliche Beziehung von Körper, Gehirn und der äußeren Umgebung (56). Seit den 1990er-Jahren wurden Entrainmenteffekte im Bereich der neurologischen Rehabilitation, im Rahmen von rhythmusgestütztem Motoriktraining bei neurologischen Patienten wie Gang- und Armtraining bei Schlaganfall- und Parkinson-Patienten, bei Patienten mit traumatischen Hirnverletzungen und zerebraler Lähmung, genutzt und untersucht (67-69). Beim RAS-Training verursachen neuronale Mechanismen eine enge Wechselwirkung zwischen dem auditorischen und dem motorischen System: Die Raten mit denen die auditorischen Neuronen feuern, prägen die Feuerraten des motorischen Systems. Dadurch bereitet das auditive System das motorische System darauf vor, synchron zum gehörten Rhythmus zu agieren (47). Diese Vorbereitung führt zu einer Handlungsbereitschaft, welche verschiedene Schritte der motorischen Ausführung wie Bewegungsinitiierung, Timing und Geschwindigkeit beeinflussen. Bereits in den späten 1960erJahren haben Paltsev und Elner (46) und Rossignol und Jones (57) untersucht, wie ein Metronom-Schlag Muskelaktivierungsmuster verändert. Alle Sinne, der visuelle, der taktile oder der olfaktorische Sinn haben unterschiedliche Auflösungen für bestimmte Parameter, die Wahrnehmung leiten. Bezüglich des Eintrainmenteffekts, 
sich synchron zu einer externen Zeitreferenz zu bewegen, kann mit akustischen Signalen die höchste Auflösung in der zeitlichen Präzision der Bewegungsausrichtung zu einem Cue erreicht werden (21).

\section{Möglichkeiten}

für musiktherapeutische Anwendungsfelder und Forschung

Angesichts der starken Zunahme von Technologien, die in der Rehabilitation ihren Einsatz finden, ergeben sich auch für Musik neue Anwendungs- und Untersuchungsmöglichkeiten. Geräte wie Rehabilitationsroboter, die oftmals mit hochauflösender Sensorik ausgestattet sind, erlauben Daten zum Therapieverlauf zu erheben. Auf Basis dieser Daten, kann die Therapie personalisiert angepasst werden und mittels effektiver Strategien dynamisch auf den Rehabilitationsprozess einwirken. Musik könnte als Teil solch einer technischen Umgebung eine interessante Rolle einnehmen: Man könnte virtuelle Instrumente nutzbar machen, Musik im Hintergrund sowie als Echtzeitfeedback einsetzen. Potentielle Effekte von Musik könnten sich in Stimmung, Motivation, Compliance, Trainingsausdauer sowie in funktionalen Verbesserungen zeigen. Lange hat die musiktherapeutische Forschung auf solche Daten und Möglichkeiten gewartet. Es gilt sie nun zu nutzen und differenzierte Schlussfolgerungen aus den Ergebnissen zu erzielen, die der Rehabilitation neurologischer Patienten zuträglich sind.

Seit Ende der 1980er-Jahre besteht ein zunehmendes Forschungsinteresse von Medizin, Technik und Wirtschaft an der Wirksamkeit von Rehabilitationsrobotern $(6,55,41)$. Dieses Phänomen geht einher mit einer steigenden Zahl neuer Geräte, einer Vielzahl von Forschungsprojekten in diesem Bereich und der Integration von Rehabilitations-Robotern im klinischen Umfeld $(33,50,52)$. Diese Systeme entsprechen einem wachsenden Bedarf an effektiver Behandlung, der von immer mehr chronisch kranken Patienten in einer alternden Gesellschaft einschließlich Schlaganfallopfern gefordert wird (19). In den westlichen Industrieländern sind beispielsweise $2 \%$ bis $5 \%$ aller Gesundheitskosten auf Schlaganfall zurückzuführen (19). Therapeutische Techniken benötigen eine Optimierung der Behandlungsqualität und ihrer Wirksamkeit. Gleichzeitig ist es sehr wichtig, neue Daten über den Verlauf der Rehabilitation zu sammeln, um prädiktive Daten zu generieren. Diese Daten können genutzt werden, um die Akteure im Gesundheitswesen über nachhaltige Präventions- und Behandlungstechniken zu informieren. Die konventionelle Therapie ist kosten- und arbeitsintensiv. Selbst wenn Robotersysteme zu diesem Zeitpunkt noch teuer sind, ist davon auszugehen, dass die Preise, je nach den therapeutischen Eigenschaften der Roboter und der Dauer ihrer Nutzung, sinken werden. Ein solcher Preisrückgang könnte darüber hinaus mit einer effektiven Integration der Robotertherapie verbunden sein, die das konventionelle klinische Umfeld erweitert $(6,37)$. Da Rehabilitationsroboter eine intelligente adaptive therapeutische Umgebung bieten, die in Einzel- und Gruppensettings einsetzbar ist, kann die Robotertherapie möglicherweise klinische Abläufe stark verändern, indem sie die Therapieintensität erhöht und gleichzeitig die manuelle Therapiezeit reduziert: Patienten können eigenständig in therapiefreien Zeiten, wie beispielsweise am Wochenende trainieren. Außerdem können verschiedene Roboter im selben Raum platziert werden. So könnten Patienten in einem Mehrspieler-Modus als Gruppe trainieren oder im Zirkeltraining mit verschiedenen Geräten arbeiten. Auch in der Gruppe könnte ein Roboter ein qualitativ hochwertiges Training gewährleisten, während die soziale Interaktion gefördert wird. Es konnte gezeigt werden, dass soziale Interaktion die Motivation erhöht, was ein sehr wichtiger Faktor für die Rehabilitationsergebnisse ist $(18,45)$. Darüber hinaus können schwer betroffene Patienten, die sich überhaupt nicht bewegen können, mit Hilfe von Robotern ihren Körper mit repetitiven Bewegungsübungen mobilisieren lassen. Diese Patientenpopulation könnte mit hoher Intensität und hoher Wiederholungsrate trainieren, was mit manueller Therapie nicht möglich ist.

Im Rahmen der Schlaganfallrehabilitation wurden Roboter speziell für das Gang- training, für die obere Extremität, für das Handgelenk und die Hände entwickelt. Bei der Rehabilitation nach einem Schlaganfall gilt die robotergestützte Rehabilitation als besonders relevant für Patienten mit schweren und chronischen Symptomen $(33,52)$. Für diese Patienten ist es wichtig, hochfrequent, intensiv und repetitiv zu trainieren. Robotertraining kann diesen Anforderungen gerecht werden: Robotersysteme bieten ein hochintensives, sich stark wiederholendes Training in verschiedenen Modi von passiv über assistiv bis hin $\mathrm{zu}$ aktivem Training mit Spielen, die Patienten dazu anregen, länger zu trainieren als bei anderen Aufgaben $(3,33,52)$. Aspekte wie motorische Lerntheorien, gut untersuchte Therapieeffekte wie hohe Intensität, hohe Bewegungswiederholungen oder Fehlerrückmeldung lassen sich leicht in Robotertherapiekonzepte übersetzen (35). Darüber hinaus können Motivationsaspekte in diese Systeme integriert werden. Hierfür sind insbesondere Spiele oder in Zirkeltrainingsszenarien im MehrspielerModi geeignet $(13,45)$. Meistens ermöglichen robotische Therapiesysteme über längere Zeiträume eine objektive und hochauflösende Messung der Performance. Dies kann genutzt werden, um Trainingsstrategien kontinuierlich zu verfeinern, den $\mathrm{Pa}$ tienten über Fortschritte zu informieren, und kann als wertvolle Datenbasis für klinische Untersuchungen zu trainingsinduzierten Effekten dienen.

In Zukunft wird die Anzahl der Rehabilitationsroboter in der Neurorehabilitation zunehmen (50). Dies geht einher mit Verbesserungen des Roboters in Richtung Softrobotik, einem höheren Grad an wahrgenommener Transparenz der tragbaren Robotik, neuen virtuellen Umgebungen mit adaptiven Trainingsalgorithmen, Fortschritten bei verfeinerten Steuerungsstrategien und neuen technologischen Kombinationen wie Robotern mit Gehirn-Computer-Schnittstellen, neuen Sensoren oder Displays wie 3D-Brillen $(5,34)$.

Musik ist als Hintergrund von virtuellen Trainingsumgebungen auch bei Rehabilitationsrobotern weit verbreitet. Auch neue Trainingsumgebungen werden akustische Elemente als Teil der virtuellen Szenarien enthalten. Neben Schlaganfall-Patienten widmet sich die Rehabilitationsrobotik ei- 
nem breiteren Spektrum von Patientengruppen, darunter Patienten mit Multipler Sklerose, Parkinson-Krankheit, Querschnittslähmung, zerebraler Lähmung, fokaler Dystonie, mit Amputationen oder anderen bewegungsbedingten Störungen. Daher müssten die Auswirkungen von Klang und Musik in der Robotertherapie immer auf die spezifische Patientenpopulation untersucht werden $(11,53)$. Dementsprechend ist eine systematische Untersuchung der Auswirkungen von Sound- und Musikanwendungen in maschinellen Neuro-Rehabilitationssystemen erforderlich, die negative Nebenwirkungen verhindert, Therapieeffekte verstärkt und Informationen über effektive Soundanwendungen für Roboter liefert. Expertenperspektiven aus Neurologie, Technik, Musikwissenschaft, Musiktherapie, Bewegungswissenschaft und Psychologie könnten zusammenarbeiten, um eine solche Wissensbasis zu schaffen.

Unterschiedliche Rollen von Musik und Klang könnten in zukünftigen Studien zu robotergestütztem Training und Musik berücksichtigt werden: Einerseits die Rolle von Musik als stimulierende Umgebungserweiterung, andererseits in der Rolle einer zusätzlichen Feedback-Modalität oder beides in Kombination. Nach van Vugt bezieht sich der Begriff „Stimulation“ auf einen externen Stimulus, der während einer Aktivität präsentiert wird, aber nicht direkt mit einer Aktion verbunden ist. Im Gegensatz dazu kann „Feedback“ als Signal definiert werden, das in direktem Zusammenhang mit einer ausgeführten Bewegung steht (72). Dementsprechend können Musik und Klang als Stimulationswerkzeug zur Erweiterung des Robotertrainings mit unterschiedlicher Hintergrundmusik, RAS oder in Kombination mit Sprache erforscht werden. Feedback könnte in Richtungen wie Fehlerrückmeldung, adaptives, korrigierendes, kontinuierliches oder kurzes Feedback untersucht werden, um zusätzliche Informationen über eine Bewegung zu liefern, interaktive Klangerlebnisse zu ermöglichen und die Wahrnehmung, Koordination und Bewegungsleistung zu fördern und die Effektivität des motorischen Lernens zu erhöhen. Musik wird oft als motivierend beschrieben und mit der gleichen Absicht auf Robotertherapien ange- wandt. Dementsprechend wäre es wichtig, besser $\mathrm{zu}$ verstehen, warum und welche Musik motiviert und wie sie zur Verfügung gestellt werden kann, um die Motivation effektiv zu steigern. Dies könnte gut in technische Umgebungen untersucht werden, in denen Musik im Hintergrund wiedergegeben wird (Stimulation) oder in denen ein Robotersystem zur aktiven Musikwiedergabe verwendet wird (Feedback). Bei aktivem Musizieren kann der Roboter auch als Controller für virtuelle Instrumente dienen, um Musik zu erzeugen oder Musik zu dirigieren. Dieser aktive Ansatz könnte aktuelle Motivationsstrategien erweitern, die darauf abzielen, Patienten mit Spielen im Einzelspieler-Modus und im Wettbewerbs- oder Kooperationsmodus in einem Mehrspieler-Modus zu motivieren (45). Im Gegensatz zu einem Spielerlebnis, bei dem der Patient ein Spiel gewinnt oder verliert (extrinsische Motivation), kann die Erzeugung oder Modulation von Musik möglicherweise gezielter intrinsische Antriebe stimulieren. Das Komponieren von Musik könnte ein prozessorientiertes Erlebnis bieten, bei dem es nicht endpunktorientiert um das Gewinnen eines Spiels geht (51). Musik kann auch zusammen mit einem anderen Patienten oder einem Therapeuten in einem Mehrspieler-Modus erstellt werden. Eine robotergestützte musikalische Interaktionsplattform könnte die Motivation, die soziale Interaktion sowie die Funktion fördern. Darüber hinaus könnte eine akustische Benutzeroberfläche eine grafische Benutzeroberfläche ersetzen. Diese kurze Liste möglicher Rollen von Musik und Sound für das Robotertraining zeigt, dass in diesem Bereich große Chancen für musiktherapeutische Forschung sowie neue Anwendungsfelder für Musik in der Rehabilitation entstehen. Musik und Klang haben Potenzial in diesem Kontext Funktion, Motivation und Kognition positiv zu stimulieren. Als langfristiges Ziel dieses Forschungsfeldes könnte die Entwicklung eines Werkzeugkastens stehen, der Auswirkungen von Musik- und Klangumgebungen auf Funktion, Motivation, Kognition und soziale Aspekte für spezifische Patientengruppen definiert.
Interessenkonflikt

Es besteht kein Interessenkonflikt.

\section{Literatur}

1. Amengual JL, Rojo N, Veciana de las Heras M, Marco-Pallarés J, Grau-Sánchez J, Schneider S et al. Sensorimotor plasticity after music-supported therapy in chronic stroke patients revealed by transcranial magnetic stimulation. PLOS ONE 2013; 8: 1-10.

2. Altenmüller EO, Schlaug G. Apollo's gift: new aspects of neurologic music therapy. Progr Brain Res 2015; 217: 237-252.

3. Balasubramanian S, Klein J, Burdet E. Robot-assisted rehabilitation of hand function. Curr Opin Neurol 2010; 23: 661-670.

4. Bangert M, Schlaug G. Specialization of the specialized in features of external human brain morphology. Eur J Neurosci 2006; 24: 1832-1834.

5. Bianchi L, Babiloni F, Cincotti F, Arrivas M, Bollero P, Marciani MG. Developing wearable bio-feedback systems: a general-purpose platform. IEEE Trans Neural Syst Rehab Eng 2003; 11: 117-119.

6. Blank AA, French JA, Pehlivan AU, O'Malley MK. Current trends in robot-assisted upper-limb stroke rehabilitation: promoting patient engagement in therapy. Curr Physic Med Rehab Rep 2014; 2: 184-195.

7. Blood AJ, Zatorre RJ. Intensely pleasurable responses to music correlate with activity in brain regions implicated in reward and emotion. Proc Nat Acad Sci 2001; 98: 11818-11823.

8. Bradt J, Magee WL, Dileo C, Wheeler BL, Mc Gilloway E. Music therapy for acquired brain injury. Cochrane Database Syst Rev 2010; 7: 1-44.

9. Burger B, Thompson MR, Luck G, Saarikallio S, Toiviainen P. Influences of rhythm- and timbre-related musical features on characteristics of musicinduced movement. Front Psychol 2013; 4: 1-10.

10. Chanda ML, Levitin DJ. The neurochemistry of music. Trends Cog Sci 2013; 17: 179-193.

11. Chen Y, Howard AM. Effects of robotic therapy on upper-extremity function in children with cerebral palsy: A systematic review. Dev Neurorehabil 2014; 19: 64-71.

12. Cross I. Music as a biocultural phenomenon. Ann New York Acad Sci 2003; 999: 106-111.

13. Dean C. Group task-specific circuit training for patients discharged home after stroke may be as effective as individualised physiotherapy in improving mobility. J Physiother 2012; 58: 269-271.

14. Eschrich S, Münte TF, Altenmüller EO. Unforgettable film music: the role of emotion in episodic long-term memory for music. BioMed Central Neuroscience 2008; 9: 1-7.

15. Friedman N, Chan V, Reinkensmeyer AN, Beroukhim A, Zambrano GJ, Bachman M, Reinkensmeyer DJ. Retraining and assessing hand movement after stroke using the MusicGlove: comparison with conventional hand therapy and isometric grip training. J NeuroEng Rehabil 2014; 11: 1-14.

16. Gärtner H, Minnerop M, Pieperhoff P, Schleicher A, Zilles K, Altenmüller EO, Amunts K. Brain morphometry shows effects of long-term musical 
practice in middle-aged keyboard players. Front Psychol 2013; 4: 1-13.

17. Gebauer L, Vuust P. Music interventions in healthcare. White Paper 2014: 1-66.

18. Hesse S, Hess A, Werner C, Kabbert N, Buschfort R. Effect on arm function and cost of robot-assisted group therapy in subacute patients with stroke and a moderately to severely affected arm: a randomized controlled trial. Clin Rehabil 2014; 28: 637-647.

19. Heuschmann PU, Busse O, Wagner M, Endres A, Villringer $J$ et al. Schlaganfallhäufigkeit und Versorgung von Schlaganfallpatienten in Deutschland. Akt Neurol 2010; 37: 333-340.

20. Huron D. Sweet anticipation. Music and the psychology of expectation. Cambridge: MIT Press 2006.

21. Hove MJ, Fairhurst MT, Kotz SA, Keller PE. Synchronizing with auditory and visual rhythms: an fMRI assessment of modality differences and modality appropriateness. NeuroImage 2013; 67: 313-321.

22. Hyde KL, Lerch J, Norton A, Foregard M, Winner E, Evans AC, Schlaug G. The effects of musical training on structural brain development: a longitudinal study. Ann New York Acad Sci 2009; 1169: 182-186.

23. Hyde KL, Lerch J, Norton A, Forgeard M, Winner E, Evans AC, Schlaug G. Musical training shapes structural brain development. J Neurosci Neuroscience 2009; 29: 3019-3025.

24. Iversen JR, Repp BH, Patel AD. Top-down control of rhythm perception modulates early auditory responses. Ann New York Acad Sci 2009; 1169: 58-73.

25. Jacobsen J, Stelzer J, Fritz TH, Chetelat G, La Joie $\mathrm{R}$, Turner R. Why musical memory can be preserved in advanced Alzheimer's disease. Brain 2015; 138: 1-13.

26. Jäncke L. Music, memory and emotion. J Biol 2008; 7: 7-21.

27. Juslin PN. From everyday emotions to aesthetic emotions: towards a unified theory of musical emotions. Phys Life Rev 2013; 10: 235-266.

28. Juslin PN What does music express? Basic emotions and beyond. Front Psychol 2013; 4: 1-15.

29. Kamioka H, Tsutani K, Yamada M, Park H, Okuizumi $\mathrm{H}$, Tsuruoka $\mathrm{K}$ et al. Effectiveness of music therapy: a summary of systematic reviews based on randomized controlled trials of music interventions. Patient Prefer Adherence 2014; 8: 727-754.

30. Keller PE, Rieger M. Musical movement and synchronization. Music Perception 2009; 26: 397-400.

31. Kirschner S, Tomasello M. Joint music making promotes prosocial behavior in 4-year-old children. Evolution and Human Behavior 2010; 31: 354-364.

32. Koelsch S. Towards a neural basis of music perception - A review and updated model. Front Psychol 2011; 2: 1-20.

33. Kwakkel G, Kollen BJ, Krebs HI. Effects of robotassisted therapy on upper limb recovery after stroke: a systematic review. Neurorehabil Neural Repair 2008; 22: 111-121.

34. Laschi C, Cianchetti M. Soft robotics: new perspectives for robot bodyware and control. Front Bioeng Biotechnol 2014; 2: 1-5.
35. Loureiro RCV, Harwin WS, Nagai K, Johnson M. Advances in upper limb stroke rehabilitation: a technology push. Med Biol Eng Comput 2011; 49: 1103-1118.

36. MacDonald R. Music, health, and well-being: A review. Int J Qual Stud Health Well-being 2013; 8: $1-13$.

37. Maciejasz P, Eschweiler J, Gerlach-Hahn K, Jansen-Troy A, Leonhardt S. A survey on robotic devices for upper limb rehabilitation. J NeuroEng Rehabil 2014; 11: 1-29.

38. Maclean N, Pound P, Wolfe C, Rudd A. Qualitative analysis of stroke patients' motivation for rehabilitation. BMJ 2000; 321: 1051-1054.

39. Maes P, Wanderley MM, Palmer C. The role of working memory in the temporal control of discrete and continuous movements. Exp Brain Res 2014; 233: 263-273.

40. Malcolm MP, Massie C, Thaut M. Rhythmic auditory-motor entrainment improves hemiparetic arm kinematics during reaching movements: a pilot study. Top Stroke Rehabil 2009; 16: 69-79.

41. Mehrholz J, Platz T, Kugler J, Pohl M. Electromechanical and robot-assisted arm training for improving arm function and activities of daily living after stroke. Cochrane Database Syst Rev 2008; 4: $1-34$.

42. Merker BH, Madison GS, Eckerdal P. On the role and origin of isochrony in human rhythmic entrainment. Cortex 2009; 45: 4-17.

43. Münte TF, Altenmüller EO, Jäncke, L. The musician's brain as a model of neuroplasticity. Nat Rev Neurosci 2002; 3: 473-478.

44. Nieminen S, Istok E, Brattico E, Tervaniemi $M$, Huotilainen M. The development of aesthetic responses to music and their underlying neural and psychological mechanisms. Cortex 2011; 47: 1138-1146.

45. Novak D, Nagle A, Keller U, Riener R. Increasing motivation in robot-aided arm rehabilitation with competitive and cooperative gameplay. J NeuroEng Rehabil 2014; 11: 1-15.

46. Paltsev EI, Elner AM. Change in the functional role of spinal segments subjected to acoustic stimulation and its role in involuntary movement. Biofizika 1967; 12: 1064-1070.

47. Patel AD. The evolutionary biology of musical rhythm: Was Darwin wrong. PLoS Biol 2014; 12: 1-6.

48. Paul S, Ramsey D. The effects of electronic musicmaking as a therapeutic activity for improving upper extremity active range of motion. Occupational Therapy International 1998; 5: 223-237.

49. Phillips-Silver J, Aktipis CA, Bryant GA. The ecology of entrainment: Foundations of coordinated rhythmic movement. Music Perception 2010; 28: 3-14.

50. Poli P, Morone G, Rosati G, Masiero S. Robotic technologies and rehabilitation: new tools for stroke patients' therapy. Biomed Res Int 2013; 2013: 153872

51. Prahalad CK, Ramaswamy V. Co-creation experiences: The next practice in value creation. J of Interactive Marketing 2014; 18: 5-14.

52. Prange GB, Jannink MJA, Groothuis-Oudshoorn CGM, Hermens HJ, Ijzerman MJ. Systematic review of the effect of robot-aided therapy on re- covery of the hemiparetic arm after stroke. J Rehabil Res Dev 2006; 43: 171-184

53. Quinn L, Busse M, Dal Bello-Haas V. Management of upper extremity dysfunction in people with Parkinson disease and Huntington disease: facilitating outcomes across the disease lifespan. J Hand Ther 2014; 26: 148-154.

54. Raglio A, Attardo L, Gontero G, Rollino S, Groppo E, Granieri E. Effects of music and music therapy on mood in neurological patients. World J Psychiatry 2015; 5: 68-78.

55. Rosati G, Oscari F, Spagnol S, Avanzini F, Masiero S. Effect of task-related continuous auditory feedback during learning of tracking motion exercises. J NeuroEng Rehabil 2012; 9: 1-13.

56. Ross JM, Balasubramaniam R. Physical and neural entrainment to rhythm: human sensorimotor coordination across tasks and effector systems. Front Human Neurosci 2014; 8: 1-6.

57. Rossignol S, Jones GM. Audio-spinal influence in man studied by the H-reflex and its possible role on rhythmic movements synchronized to sound. Electroencephalogr Clin Neurophysiol 1967; 41: 83-92.

58. Repp BH, Penel A. Rhythmic movement is attracted more strongly to auditory than to visual rhythms. Psychol Res 2004; 68: 252-270.

59. Sammler D, Grigutsch M, Fritz T, Koelsch S. Music and emotion: electrophysiological correlates of the processing of pleasant and unpleasant music. Psychophysiology 2007; 44: 293-304.

60. Särkämö T, Soto D. Music listening after stroke: beneficial effects and potential neural mechanisms. Ann New York Acad Sci 2012; 1252: 266-281.

61. Särkämö T, Tervaniemi M, Laitinen S, Forsblom A, Soinila S, Mikkonen $\mathrm{M}$ et al. Music listening enhances cognitive recovery and mood after middle cerebral artery stroke. Brain 2008; 131: 866-876.

62. Schneider S, Schönle PW, Altenmüller EO, Münte TF. Using musical instruments to improve motor skill recovery following a stroke. J Neurol 2007; 254: 1339-1346.

63. Speth F. The role of sound in robot-assisted hand function training post-stroke. Diss Rehabilitationswissenschaften. Humboldt Universität zu Berlin 2017.

64. Taheri H, Rowe JB, Gardner D, Chan V, Gray K, Bower $\mathrm{C}$ et al. Design and preliminary evaluation of the FINGER rehabilitation robot: controlling challenge and quantifying finger individuation during musical computer game play. J NeuroEng Rehabil 2014; 11: 1-10.

65. Thaut MH. Music as therapy in early history. Progr Brain Res 2015; 217: 143-158.

66. Thaut MH, Kenyon GP, Hurt CP, McIntosh GC Hömberg V. Kinematic optimization of spatiotemporal patterns in paretic arm training with stroke patients. Neuropsychologia 2002; 40: 1073-1081.

67. Thaut MH, McIntosh GC, Hömberg V. Neurobiological foundations of neurologic music therapy: rhythmic entrainment and the motor system. Front Psychol 2015; 5: 1-6.

68. Thaut MH, McIntosh GC, Rice RR, Miller RA, Rathbun J, Brault JM. Rhythmic auditory stimulation in gait training for Parkinsons disease patients. Mov Dis 1996; 11: 193-200. 
69. Thaut MH, McIntosh GC, Prassas SG, Rice RR. Effect of rhythmic auditory cuing on temporal stride parameters and EMG. Patterns in hemiparetic gait of stroke patients. Neurorehabil Neural Repair 1993; 7: 9-16.

70. Van der Hejden MJ, Oliai AS, van Dijk M, Jeekel J, Hunink MG. The effects of perioperative music interventions in pediatric surgery: A systematic review and meta-analysis of randomized controlled trials. PLoS ONE 2015; 10: 1-11.
71. Van Vugt FT. Sounds on time: auditory feedback in motor learning, re-learning and over-learning of timing regularity. Neuroscience. Université Claude Bernard- Lyon I. 2013.

72. Van Vugt FT, Kafczyk T, Kuhn W, Rollnik JD, Tillmann B, Altenmüller EO. The role of auditory feedback in music-supported stroke rehabilitation: A single- blinded randomised controlled intervention. Restor Neurol Neurosci 2016; 34 297-311.
73. Vuilleumier P, Trost W. Music and emotions: from enchantment to entrainment. Ann New York Acad Sci 2015; 1337: 212-222.

74. Wan CY, Schlaug G. Music making as a tool for promoting brain plasticity across the life span. Neuroscientist 2010; 16: 566-577. 


\section{Zertifizierte Fortbildung}

\section{Fragen zum Thema „Musik in der Neurorehabilitation nach einem Schlaganfall"}

\author{
1. Seit wann findet Musik ihren Einsatz \\ als therapeutisches Werkzeug? \\ a) Seit Beginn dieses Jahrhunderts. \\ b) Seit der Antike. \\ c) Seit der Erfindung des Klaviers. \\ d) Seit 1840. \\ e) Seit 1967.
}

\section{Was bedeutet „RAS"?}

a) Rhythmische Aphasie Sitzung.

b) Rhythmisch akustische Stimulation.

c) Regenerative akustische Stimulation.

d) Rehabilitation der Artikulation und Stimme.

e) Rhythmisch akustische Singübung.

3. Seit wann wird der Effekt eines Metronomstimulus auf Muskelaktivität untersucht?

a) Seit der Erfindung des Metronoms.

b) Seit 1967.

c) Seit der Etablierung von „RAS" als Therapiemethode.

d) Seitdem es EMG-Messgeräte gibt.

e) Seit der Erfindung isometrischen Trainings.

4. Welche Bereiche musiktherapeutischer Anwendungen sind im Rahmen von Schlaganfalltherapie für die Motorik wissenschaftlich als wirksam belegt?

a) Musikhören.

b) Rhythmisch akustische Stimulation.

c) Tanzen.

d) Komposition.

e) Gruppensingen.
5. Unter welchen Bedingungen erinnert man sich, laut einer Untersuchung von Jäncke et al., am besten an ein einmalig gehörtes, noch unbekanntes Musikstück?

a) Wenn das Stück subjektiv als stark emotional wahrgenommen wurde.

b) Wenn das Stück subjektiv konzentriert gehört wurde.

c) Wenn das Stück gesungene Partien enthält.

d) Wenn das Stück große Lautstärkeunterschiede beinhaltet.

e) Wenn das Stück Angst ausgelöst hat.

6. Welche Instrumente wurden in klinisch evaluierten musiktherapeutischen Settings in der Schlaganfalltherapie bereits untersucht?

a) Gitarre/ Geige

b) Keyboard/ Drumpad

c) Blockflöte/ Querflöte

d) Trompete/ Posaune

e) Harfe/ Cembalo

7. Welches Netzwerk wird im Gehirn bei Musikhören insbesondere aktiviert?

a) Motorisches Netzwerk.

b) Auditives Netzwerk.

c) Sensomotorisch-auditives Netzwerk.

d) Gedächtnisnetzwerk.

e) Auditiv-visuelles Netzwerk.

8. Wodurch unterscheidet sich ein Nichtmusikergehirn von einem Gehirn eines Musikers, der bereits seit seiner frühen Kindheit regelmäßig ein Instrument erlernt hat?

a) Unterschiede in der Größe des Gehirns.

b) Unterschiede in der Ausprägung des limbischen Systems.

c) Unterschiede in der Größe des visuellen Kortex.

d) Unterschiede im Gehirn und kortikospinalen Trakt.

e) Unterschiede im Sprachareal.

\section{CME-Fortbildung online}

Die Teilnahme an dieser durch die Ärztekammer Nordrhein zertifizierten CME-Fortbildung ist für 12 Monate ausschließlich online möglich. Zur Anmeldung gehen Sie bitte auf http://cme.thieme.de. Dort erfahren Sie auch den genauen Teilnahmeschluss. Es ist immer nur eine Antwort pro Frage zutreffend. Als Leser der Nervenheilkunde nehmen Sie kostenlos am CME-Programm teil. Je nach CME-Fortbildung erhalten Sie bis zu vier Fortbildungspunkte. Weitere Informationen zur Anmeldung und Registrierung finden Sie unter http://cme. thieme.de. Sie erhalten bei inhaltlichen und technischen Fragen tutoriellen Support. VNR 2760512018154652895

9. Welche Vorteile könnten robotergestützte Rehabilitationssysteme im klinischen Alltag bieten?

a) Erhöhung der Trainingsintensität.

b) Beruhigung des Patienten.

c) Verbesserung des vegetativen Systems.

d) Therapeuten werden ersetzt.

e) Eine vertraute Umgebung.

10.Für welche Patientengruppe ist Musik in der robotergestützten Handrehabilitation nicht förderlich?

a) Für Patienten mit einer schweren Hemiparese mit einer ausgeprägten Spastik.

b) Für Schlaganfall-Patienten mit einer leichten Hemiparese.

c) Für junge Schlaganfall-Patienten.

d) Für Schlaganfall-Patienten mit Broca-Aphasie.

e) Für Schlaganfall-Patienten in der frühen Phase nach dem Schlaganfall. 